\title{
Study on the Impact of Urban Rail Transit on Tourism Economy in Guangdong Based on Urban Functional Network Model
}

\author{
Du Kangsheng ${ }^{1}$, Huang Xiang (Corresponding author) ${ }^{2 * a}$, Luo Weitao ${ }^{3}$, Meng Yingying $^{4}$, Zhou Yanjun $^{5}$, Li Hanwei $^{6}$ \\ ${ }^{1}$ GDUFE Hotel Management, Handan, China \\ ${ }^{2}$ GDUFE E-Commerce, Zhuhai, China \\ ${ }^{3}$ GDUFE Financial Management, Dongguan, China \\ ${ }^{4}$ GDUFE Financial, Shijiazhuang, China \\ ${ }^{5}$ GDUFE Hotel Management, Chungking, China \\ ${ }^{6}$ GDUFE Financial Management, Mianyang, China
}

\begin{abstract}
Urban rail transit is a new type of rail transit, between railway and urban rail transit, mainly used to solve the traffic problems between cities. By using the urban functional network model, this paper analyzes the complementary coefficient changes of Guangzhou, Zhuhai and Macao before and after the completion of urban rail transit in Guangdong Province. Through quantitative data, it intuitively shows the objective changes brought by urban rail transit to urban planning and development, and also brings development space for urban tourism economy, and deepens the economic ties between cities.
\end{abstract}

\section{Introduction}

\subsection{Construction status of urban railway in Guangdong Province}

Guangzhou-Zhuhai urban rail transit, referred to as Guangzhou-Zhuhai urban rail, also known as Guangzhou-Zhuhai urban railway, runs from Guangzhou South Railway Station in Guangzhou City in the north to Shunde District and Zhongshan City in Foshan City in the south to Zhuhai Station at Gongbei Port in Zhuhai City. The total length of the whole line is $177.3 \mathrm{~km}$ (including Jiangmen Branch Line and Airport Extension Line), and the design speed is $200 \mathrm{KM} / \mathrm{H}$. Guangzhou South Station to Zhuhai Station is 116 kilometers (Guangzhou South Station to Zhuhai North Station is 93 kilometers, Zhuhai North Station to Zhuhai Station is 23 kilometers), Xiaolan Station to Xinhui Station is 26.328 kilometers, Zhuhai Airport branch line is 35.3 kilometers. Jiangmen branch connects the Huicheng Street of Jiangmen Xinhui District from Xiaolan Town in Zhongshan City to Guzhen Town in Zhongshan City, across the Xijiang River.

Guangzhou-Zhuhai urban rail transit was opened on December 31, 2012. The fastest time from Guangzhou South Station to Zhuhai Station was 59 minutes, and the fastest time from Zhuhai Station to Guangzhou South Station was 58 minutes. It takes only 55 minutes for Guangzhou South Station to Xinhui Station and 55 minutes for Xinhui Station to Guangzhou South Station.

Since April 16, 2017, Guangzhou Railway Group will also run three pairs of two-way one-stop direct trains from Zhuhai to Guangzhou South, namely Guangzhou
South-Zhuhai G6333, G6335 passenger trains. Zhuhai-Guangzhou South G6334, G6336 passenger trains, Guangzhou South-Zhuhai D7265, Zhuhai-Guangzhou South D7266 passenger trains, all for about 55 minutes.

\subsection{Advantages of urban rail transit}

\subsubsection{High carrying capacity, transport capacity.}

There are 488 passengers on a harmonious train with eight carriages. Each day, there are 38 flights from Guangzhou to Zhuhai, and 38 flights from Zhuhai to Guangzhou, which can meet 3,708 passengers.

\subsubsection{Fast transportation.}

Taking Guangzhou to Zhuhai as an example, under normal weather and non- holiday conditions, the time required for driving is about 2 hours. In bad weather or holidays, the time for driving will increase by 1 to 2 hours. If taking the urban railway, the whole journey time is 1 hour, which is much less than the time used by the car. At the same time, there is no traffic congestion in the urban rail, so that the travel time of passengers can be guaranteed.

\subsubsection{Comfortable riding environment}

Urban rail train interior layout relative to passenger cars is more luxurious, comfortable seats, good driving performance, smooth operation. Due to the use of shock absorption and sound insulation, the environment 
in the carriage is quiet. The car also provides a washroom and a dining car, which meets the various needs of passengers during the ride.

\subsection{Changes in transport patterns}

In July 2016, the National Development and Reform Commission of China held a press conference to introduce the "Medium and Long Term Railway Network Planning" situation. On the basis of the original plan of "four longitudinal and four transverse" main skeleton, the national high-speed railway network increased the high-speed railway with passenger flow support, suitable standards and development needs. At the same time, it made full use of the existing railway to form a high-speed railway network with "eight longitudinal and eight transverse" main channel as the skeleton, regional connection line connection and urban railway supplement.

\subsection{Research purpose}

This paper aims to show the objective changes brought by intercity rail transit to urban planning and development directly through quantitative data, bring wider development space for the city's tourism economy, and deepen the economic relations between cities.

\section{Literature review}

The development of urban rail transit is a unique planning in the construction of Guangdong-Hong Kong-Macao Greater Bay Area. At present, there are only relevant studies on the economic impact of the development of subway, expressway, high-speed railway and railway on the region. This paper discusses the impact of various traffic types studied by previous people on the local area.

Such as Zhu Taoxing of Shijiazhuang Railway University, through the establishment of 10 tourist cities along the Beijing-Shanghai high-speed railway as the research object, the establishment of accessibility model, tourism economic contact model and coupling coordination model, respectively measure the accessibility before and after the operation of high-speed rail, tourism economic contact and the degree of coordinated development of the two, the study concluded that high-speed rail greatly compressed the space-time distance of tourist cities along the line, the accessibility increased significantly, the accessibility of tourist cities along the line increased by more than $50 \%$, of which Jining accessibility increased by $70.37 \%$.[1]The team also constructs the utility function model of tourists ' travel value based on the principle of tourists ' behavior decision-making process and utility function. The sharing rate of high-speed railway compared with ordinary railway and highway is obtained by Logit model. The regression prediction method is used to calculate the high-speed rail travel demand of tourists in Beijing-Tianjin-Hebei regions and metropolitan areas in the future. It is concluded that the resource level of tourist attractions, traffic costs, travel distance, influence of tourist destinations and traffic time are the main factors affecting tourists ' travel decisions, which constitute the basis for evaluating the value of tourists ' travel modes.[2]And scholars Zhang Yunjiao to Yuxi city ' $\mathrm{s}$ socio-economic, urban traffic and tourism development characteristics of a comprehensive combing, through the analysis of traffic crux and research tourism and urban commuting passenger flow combination of urban population travel characteristics, analysis of urban rail transit level division, put forward Yuxi city tourism rail transit network including regional fast-forward high-speed railway and national railway trunk, high-speed railway and regional rail of global communication, three-dimensional rail of internal commuting, etc., to reduce the cost of tourism by traffic lines to expand the market case.[3]

Specifically, the water traffic and land traffic coexist in Guangdong Province, the geomorphological environment and hydrological environment are complex, and the economic development of eastern and western Guangdong is different. The development of inter-city rail transit by point to area can solve the problem of coordinated development of urban agglomeration in Dawan District to a certain extent, which is similar to the conclusion of Shen Xuejun in the relevant research of transportation hub. The layout of comprehensive transportation hub is the key research problem in hub construction. The planning, design and construction of comprehensive transportation hub should take planning as the core, streamline as the leading, all parties actively coordinate, seize the main contradiction, solve the secondary contradiction, and strive to maximize the comprehensive benefit.[4]

\section{Research methods}

\subsection{Model overview and data sources}

This paper takes the evolution of urban function as a new perspective of urban connection research, and studies the urban function network of the three cities in Guangdong Province based on the above development trend and research progress of geographical urban connection, combined with the urban connection model based on the framework of urban function network analysis proposed by Liu Tao et al. This study will use the statistical yearbook of Guangdong Province in different years, the relevant statistical yearbook of other government departments, the statistical data of industry associations, the data of professional consulting companies, combined with the field survey data of various cities and departments, to calculate the complementarity coefficient between cities (FCIij).

\subsection{Calculation method}

$$
L Q_{i k}=\frac{E_{\mathrm{ik}} / E_{\mathrm{i}}}{E_{\mathrm{k}} / E}
$$


LQik represents the location quotient of the catering sector in the research scope of city I; Eik represents the number of employees of the catering sector in city $\mathrm{i} ; \mathrm{Ei}$ represents the total number of employees of the catering sector in the research scope; Ek represents the total number of employees of the catering sector in the research scope; E represents the total number of employees of various industries in the research scope. In the Krugman index model, the sum of the absolute value of regional quotient difference of each industry sector between cities is taken as the functional complementarity coefficient (FCIIJ) between these two cities. The larger the index is, the closer the functional connection between cities is.

$$
F C I_{i j}=\sum_{k=1}^{n}\left|L Q_{i k}-L Q_{j k}\right|
$$

Where $\mathrm{i}$ and $\mathrm{j}$ represent two different cities, FCIij represents the functional complementation coefficient of city $i$ and city $j, k$ represents the catering sector of each city, $\mathrm{n}$ represents the number of industrial sectors of each city, and LQik represents the location quotient of the catering sector of city $i$ within the research scope. The sum of the absolute value of the location quotient difference of each industry sector between cities is taken as the functional complementarity coefficient (FCIij) between these two cities. The larger the index is, the closer the functional connection between cities will be and the stronger the complementarity will be.

\section{Statistical results and research analysis}

\subsection{The statistical results}

Through relevant calculation, we obtained the functional complementarity coefficients of Guangzhou-Zhuhai, Guangzhou-Macao and Zhuhai-Macao from 2010 to 2015.

The following table:

Table 1. Urban Functional Complementarity Coefficient

\begin{tabular}{|c|c|c|c|c|c|c|c|}
\hline \multicolumn{8}{|c|}{ Urban Functional Complementarity Coefficient } \\
\hline City & Year & 2010 & 2011 & 2012 & 2013 & 2014 & 2015 \\
\hline \multicolumn{2}{|c|}{$\begin{array}{c}\text { Guangzhou\& } \\
\text { Zhuhai }\end{array}$} & 0.512249603 & 0.36744797 & 0.3267189 & 0.562056567 & 0.789341613 & 0.864538853 \\
\hline \multicolumn{2}{|c|}{$\begin{array}{c}\text { Guangzhou\& } \\
\text { Macao }\end{array}$} & 5.488053253 & 5.481319731 & 8.031842228 & 7.858890318 & 7.304348424 & 8.06225203 \\
\hline \multicolumn{2}{|c|}{ Macao \&Zhuhai } & 4.97580365 & 5.113871761 & 7.705123328 & 7.296833751 & 6.515006812 & 7.197713178 \\
\hline
\end{tabular}

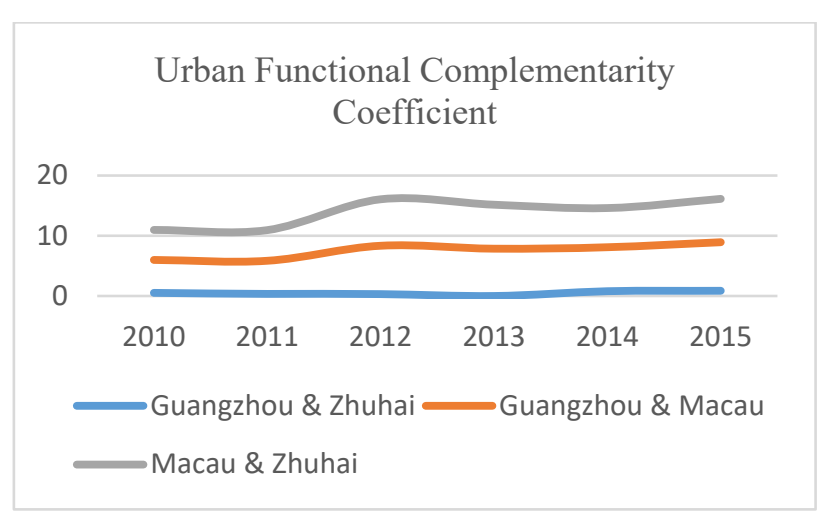

Figure 1 Urban Functional Complementarity Coefficient

\subsection{Research and analysis}

(1)

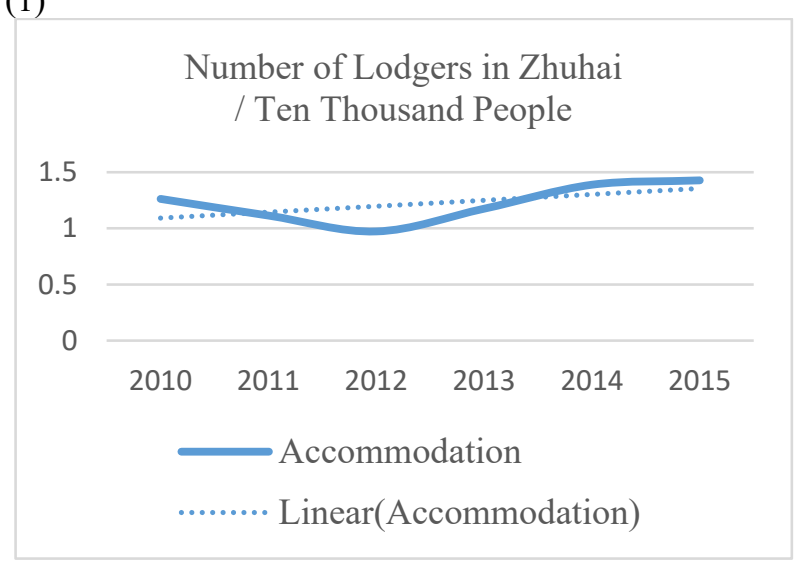

Figure 2 Number of Lodgers in Zhuhai

As can be seen from Figure 1, from 2010 to 2015, the number of lodgers in Zhuhai's accommodation industry showed a trend of decrease at first and then increase. The number of lodgers dropped to the lowest level in 2012 and began to rise at a fast speed. It is clear that the construction of the urban rail system in 2012 has helped to boost the development of the accommodation industry. 


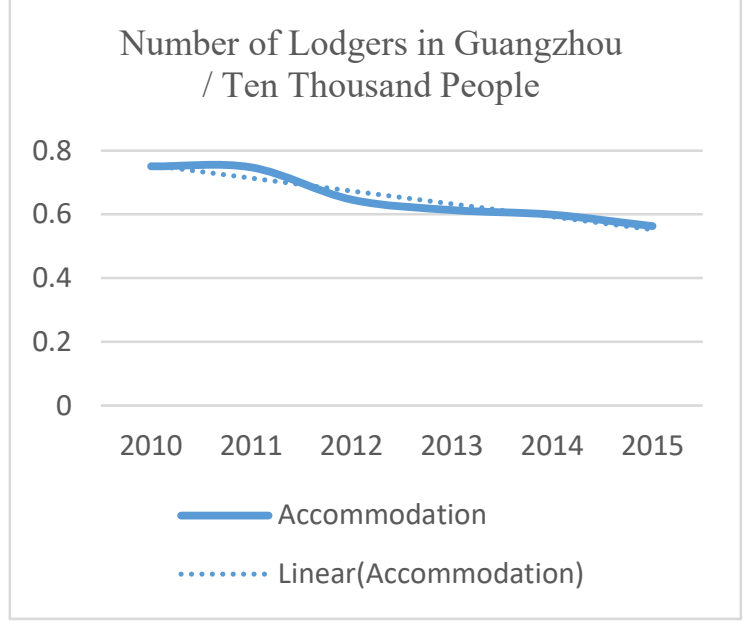

Figure 3 Number of Lodgers in Guangzhou

As can be seen from Figure 2, the number of lodgers in Guangzhou's accommodation industry decreased slightly year by year from 2010 to 2015 . But overall numbers have not fallen much. From a variety of perspectives, it may be related to the urban rail's promotion of Zhuhai, Macao and other surrounding tourism after its completion.

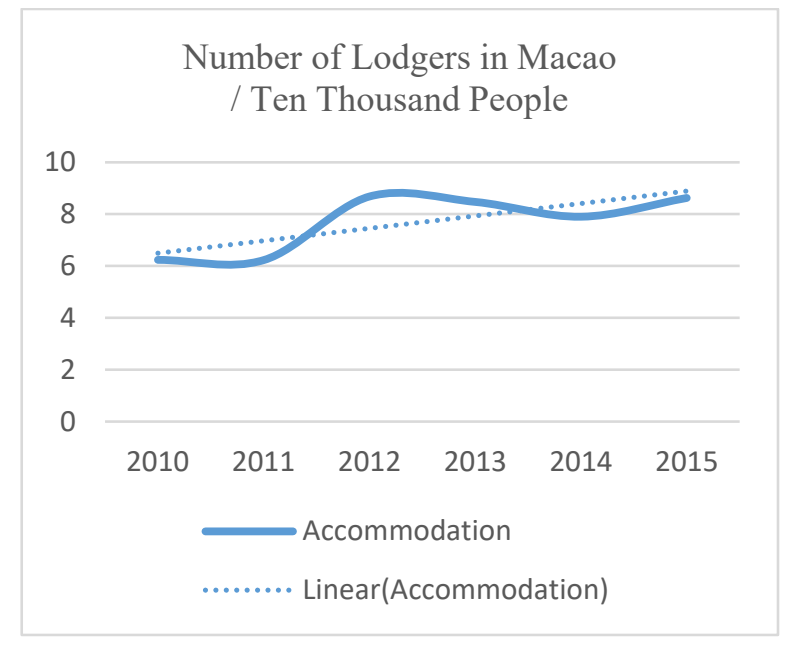

Figure 4 Number of Lodgers in Macao

As can be seen from Figure 3, the number of lodgers in Macao's accommodation industry decreased slightly from 2010 to 2011, and increased at a fast rate from 2011 to 2012. The number decreased from 2012 to 2014, and increased to the highest point in the past five years after 2014. Among them, after the opening of the urban rail in 2012 , compared with the number of lodgers before the opening of the urban rail, there was a significant increase. Therefore, we can conclude that the urban rail has a profound impact on the local accommodation industry.

General analysis: from the above overall data, the average location quotient of Guangzhou-Zhuhai is greater than 0.5 , and the average location quotient of Guangzhou-Macao and Zhuhai-Macao is greater than 5 . In general, the number of passengers between guangdong and Macao and zhuhai-Macao has increased significantly compared with that before the opening of guangdong and
Zhuhai rail. At the same time, it can also be seen that the opening of urban rail has a certain driving effect on the development of accommodation industry in the three places. It can be concluded that the opening of the urban rail will not only promote the tourism of guangzhou, zhuhai and macau, but also promote the lodging industry related to tourism.

\section{Research conclusion and development strategy}

\subsection{The research conclusion}

After rigorous investigation, analysis and scientific research, this paper draws the following conclusions:

(1) The opening of urban rail has changed the traffic pattern in the west bank of the Pearl River Delta. In 2012, the opening of the entire Guangzhou-Zhuhai Railway further extended the distance between Macao and the mainland, which makes Macau truly integrate into the Pearl River Delta's one-hour living circle and one-hour economic circle. This will greatly facilitate the integration of Guangdong, Hong Kong and Macao. It is also of great significance to the practice of "one country, two systems".

(2) The opening of urban rail has changed the way of tourism. It can be seen from the way people usually travel at present, convenient transportation for regional tourism promotion effect is often a two-way street, and the opening of the urban rail greatly convenient for outsiders to Zhuhai. It can bring more tourists to Zhuhai and also promote the travel between the five cities on the west bank of the Pearl River Delta to a large extent. With the opening of the Zhuhai section of the Guangzhou-Zhuhai Rail Line and the completion of the Hong Kong-Zhuhai-Macao Bridge, a large number of mainland residents can take the Guangzhou-Zhuhai Rail Line to Macao through the gongbei port, and a large number of Hong Kong-Macao residents can also come to Zhuhai more conveniently for leisure travel.

(3) The opening of urban rail has also changed the development trend of tourism. The continuous operation of urban rail has brought a revolution of tourism space, which is conducive to residents' travel, and innovates the traditional concepts of tourism development and marketing: inter-city tourism market integration, urban and rural tourism market compact and three-place tourism market connection. The urban rail shortens and integrates regional relations. It transforms the traditional loose-knit and conceptual cooperation in tourism development between cities into substantive urban integration and first-line cooperation. It closely integrates Zhuhai, Zhongshan, Guangzhou and other cities, and constructs the "Pearl River Delta one-hour economic circle" in real sense, which greatly shorten the geographical spatial barrier and the psychological distance of tourists, which is conducive to shorten the market space of the three places and promote the increase of tourist flow in the scenic spots. In addition, the opening of the Guangzhou-Zhuhai Railway has formed a one-hour traffic circle in the Pearl River Delta. 
(4) The opening of urban rail has an impact on the emerging tourism products. The rapidity, economy, safety, comfort and convenience of Guangzhou-Zhuhai Rail have a great influence on the travel of short-distance tourists and a strong attraction for long-distance tourists. However, the situation that the distance between the station and the destination affects the travel intention of tourists still needs to be improved.

(5) The opening of urban rail has caused a certain impact on other industries. For example, the automobile transportation industry of highway passenger transport: the urban rail will not be stuck in traffic like the car, and its running time is much shorter than that of the car, and its low price, safety and comfort, which brings great challenges to the automobile transportation market.

\subsection{The development strategy}

(1) Promote local culture and shape city image. The construction of urban cultural characteristics is an important part of the process of urbanization development, and also an important guarantee of urbanization development. Guangzhou, Zhuhai and Macao should strengthen the publicity of local culture, deepen the image of the city, and add more connotation to attract more tourists to visit.

(2) Coordinated development of points, lines and planes. "Point" means to promote the independent economic development of each region by relying on Guangzhou, Zhuhai and Macao respectively. "Lines" refers to the flow of resources gathered by various "points" along the "line" and the development of regional economic belt based on the general law of "connecting points with lines". "Planes" means that the three places are connected to form a plane, covering the surrounding cities, driving the tourism development of Zhongshan, Foshan and other cities through the Guangzhou-Zhuhai Railway, strengthening the economic ties in the nearby regions and promoting the cooperative relations between cities.

(3) Integrate tourism resources and develop tourism products. Guangzhou, a city with a long history of culture, is also the capital city of Guangdong Province. It has Baiyun Airport, one of the three major airports in China, and a number of railways connected with other provinces and cities to bring it a large number of passengers. For Zhuhai, Macao two cities, one of its pillar industries both are tourism, and its development strategy has also received the attention of the government and relevant departments. But ties between the three countries are still weak. More publicity should be given to the other two cities at the airport, high-speed rail stations, urban rail stations and we should invite some regular travel agencies to set up and provide tourism routes and tourism planning services in the three cities, so as to promote the mutual promotion and common development of the tourism economy of the three cities.

(4) Improve the public service system for tourism. First of all, it is necessary to improve the tourism information center, such as the establishment of tourism information center in the city rail to facilitate tourists to consult. Moreover, it is necessary to accelerate the improvement of convenient transportation service system, such as setting up tourism distribution service center at the exit of urban rail, improving the service function of tourism distribution, optimizing the layout of tourism bus routes, setting up special tourist lines, and building the tourism distribution channel connecting the core tourism area and the periphery of the city. Finally, establish and improve the tourism industry information platform, support the implementation of intermediary organizations in accordance with the law such as the green jade association make travel agencies, tour guides, hotels, scenic spots such as service evaluation standard, set up a regular release exposure tourism business service unhealthy or illegal information released in accordance with the law of working mechanism, support media exposure time of tourism violation, create a strong atmosphere to encourage integrity and punish dishonesty.

\section{Acknowledgments}

This paper is a phased research result of the Guangdong Provincial Education Department (No. 2019WTSCX031).

\section{References}

1. Zhu Taoxing, Ge Yong, Wang Hui. Research on the coordinated development of tourism economy between Beijing-Shanghai high-speed railway and regions along the railway [J]. Journal of Railway Engineering, 2019, 36(11): 99-102+107.

2. Zhu Taoxing, Ren Jianxin, Zhang Xueyan. Research on travel demand of Beijing-Tianjin-Hebei high-speed railway based on utility function $[\mathrm{J}]$. Journal of Railway Engineering, 2018, 35(03): 102-108.

3. Journal of Railway Engineering,2019,36(05):52-58. ZHANG Yun-jiao. Study on Tourism Rail Tranform Network Planning in Yuxi City [J]. Journal of Railway Engineering, 2019,36(05):52-58.

4. SHEN Xuejun. Discussion on the Construction of Integrated Transportation Hub [J]. Journal of Railway Engineering, 2014,31(10):79-83. 